\title{
A Hybrid Approach of Data-Driven and Physics-based Methods for Estimation and Prediction of Fatigue Crack Growth
}

\author{
Hyeon Bae Kong ${ }^{1}$, Soo-Ho Jo ${ }^{2}$, Joon Ha Jung ${ }^{3}$, Jong M. Ha ${ }^{4}$, Yong Chang Shin ${ }^{5}$, Heonjun Yoon ${ }^{6}$, Kyung Ho Sun ${ }^{7}$, Yun-Ho \\ $\mathrm{Seo}^{8}$, and Byung Chul Jeon ${ }^{9}$ \\ 1,2,5,6 Department of Mechanical and Aerospace Engineering, Seoul National University, Seoul, 08826, Republic of Korea \\ vividsunmi@snu.ac.kr \\ jsh1201@snu.ac.kr \\ yongchang88@snu.ac.kr \\ heonjun@snu.ac.kr \\ 3,7,8 Department of System Dynamics, Korea Institute of Machinery \& Materials, Daejeon, 34103, Republic of Korea \\ joonha@kimm.re.kr \\ sunkh@kimm.re.kr \\ yhseo@kimm.re.kr \\ ${ }^{4}$ Center for Safety Measurement, Korea Research Institute of Standard and Science, Daejeon, 34113, Republic of Korea \\ jmha@kriss.re.kr \\ ${ }^{9} 18$ th Fighter Wing, Republic of Korea Air Force, Gangneung, Republic of Korea \\ jeonbc@airforce.mil.kr
}

\begin{abstract}
This paper aims to develop a hybrid method to estimate the fatigue crack growth of an aluminum lap joint specimen with and without Lamb wave signals. The proposed method is validated on the two validation specimens (T7 and T8), using the training data sets of six different specimens (T1-T6). Each validation data set includes crack length estimation of few loading cycles with the given Lamb wave signals, followed by crack estimation without the signals. First, the crack length estimation using the signals for $\mathrm{T} 7$ and $\mathrm{T} 8$ sets was performed by the data-driven based method. A set of features was extracted from the preprocessed signals. Then, a random forest model was used to estimate crack lengths with grid search-based feature selection and hyper-parameter optimization. Next, different approaches were used to estimate the crack length without the signals, since T7 and T8 were tested under different loading conditions. Assuming that the homogeneous constant loading condition leads to a similar fatigue crack growth patterns, an ensemble prognostics approach with simplified particle filter-based weight update was used to predict the crack lengths of T7 specimen. In contrast, Walker's equation model-based approach was chosen for $\mathrm{T} 8$ specimen as it was tested under a different loading condition. Considering the uncertainties of the model parameters, Walker's equation models were generated by Monte Carlo methods. The average of generated
\end{abstract}

models were used to predict the remaining crack lengths of T8 specimen. The proposed method led to Top 3 in 2019 PHM Conference Data Challenge. 